\title{
Quantitative Characterization of Crystal Defects in Planetary Materials in a Scanning Electron Microscope (SEM)
}

\author{
Shirin Kaboli ${ }^{1}$, Pamela C. Burnley ${ }^{2}$ \\ 1,2. Geoscience Department and High Pressure Science and Engineering Center, University of Nevada \\ Las Vegas, Las Vegas, United States.
}

The flow behavior of planetary materials at high pressure and high temperature controls the dynamic processes in planets. Quantifying deformation mechanisms is necessary for establishing flow laws from experimentally deformed materials that can then be extrapolated to natural materials deformed on real time and spatial scales. There are two main crystal defects that are important for plastic deformation of polycrystalline materials: twins and dislocations which reflect the conditions and degree of plastic deformation. Therefore, quantitative characterization of these crystal defects at the nanoscale in planetary materials is of significant importance in the rock and mineral physics community [1].

Geologists have primarily used transmission electron microscopy (TEM) for nanoscale study of crystal defects in minerals. Materials scientists, however, commonly use field emission scanning electron microscopes (FE-SEM) with nanoscale spatial resolution, utilizing electron channeling contrast imaging (ECCI) and electron backscatter diffraction (EBSD) techniques to study crystal defects in bulk samples [2]. SEM on bulk samples is advantageous over TEM on thin foils due to fast and non-destructive sample preparation, a large field of view and statistically reliable results from a heterogeneously deformed microstructure of a polycrystal. In this contribution, we showcase the use of FE-SEM for quantitative characterization of crystal defects in a number of planetary materials including olivine and quartz experimentally deformed at high pressure-temperature conditions. ECCI on these materials is particularly challenging due to low channeling contrast in olivine and electron beam damage in quartz. We present novel protocols for sample preparation and microscope operation conditions to overcome these difficulties and further extend established limits of conventional SEM to characterization of crystal defects in both experimentally deformed and naturally deformed planetary materials.

We show an example of an ECCI study of experimentally deformed polycrystalline diopside $\left(\mathrm{MgCaSi}_{2} \mathrm{O}_{6}\right)$ in a Sigma Zeiss VP-SEM. The sample was hot pressed and subsequently deformed in a Griggs apparatus. Figure 1 shows the microstructure of a deformed diopside grain containing twins and subgrains. Twins are in the form of bands crossing the entire width of the grain in (a). In addition to twinning, the parent grain is divided into several subgrains of different channeling contrast relative to adjacent subgrains. Measurements on twins show several degrees of curvature in their path as they cross the subgrain boundaries as shown in (b). Figure 1c shows a closer view of twins and subgrain boundaries. Twin boundaries were decorated with edge-on dislocations indicated with arrows in (c). These edge-on dislocations were also observed on the subgrain boundaries implying the formation of these boundaries as a result of dislocation slip. A comparison of this microstructure with the microstructure of starting material shows that the mechanical twinning was the dominant deformation mechanism during deformation of this material. The results of EBSD crystal orientation mapping showed $\langle 001\rangle 180^{\circ}$ angle-axis pair of misorientation for the twin boundaries. These preliminary results show promising potential of our protocols for successful quantitative characterization of crystal defects in planetary materials in FE-SEM. 


\section{References:}

[1] D.J. Weidner, Y. Wang, G. Chen, J. Ando and M.T. Vaughan, in "Properties of Earth and Planetary Materials at High Pressure and Temperature" ed. M.H. Manghnani and T. Yagi, (American Geophysical Union, Washington, D. C.), p. 473.

[2] A.J. Wilkinson and P.B. Hirsch, Micron 28(1997), p. 279.

[3] This research was sponsored by the National Science Foundation under award NSF-EAR13613399 Additional support was provided to the second author by the National Nuclear Security Administration under the Stewardship Science Academic Alliances program through DOE Cooperative Agreement \#DE-NA0001982. Synchrotron experiments were performed at the Advanced Photon Source which is supported by DOE-BES, under Contract No. DE-AC02-06CH11357 at Sector 6BM which is supported by the Consortium for Materials Properties Research in Earth Sciences under NSF cooperative agreement EAR 06-49658.
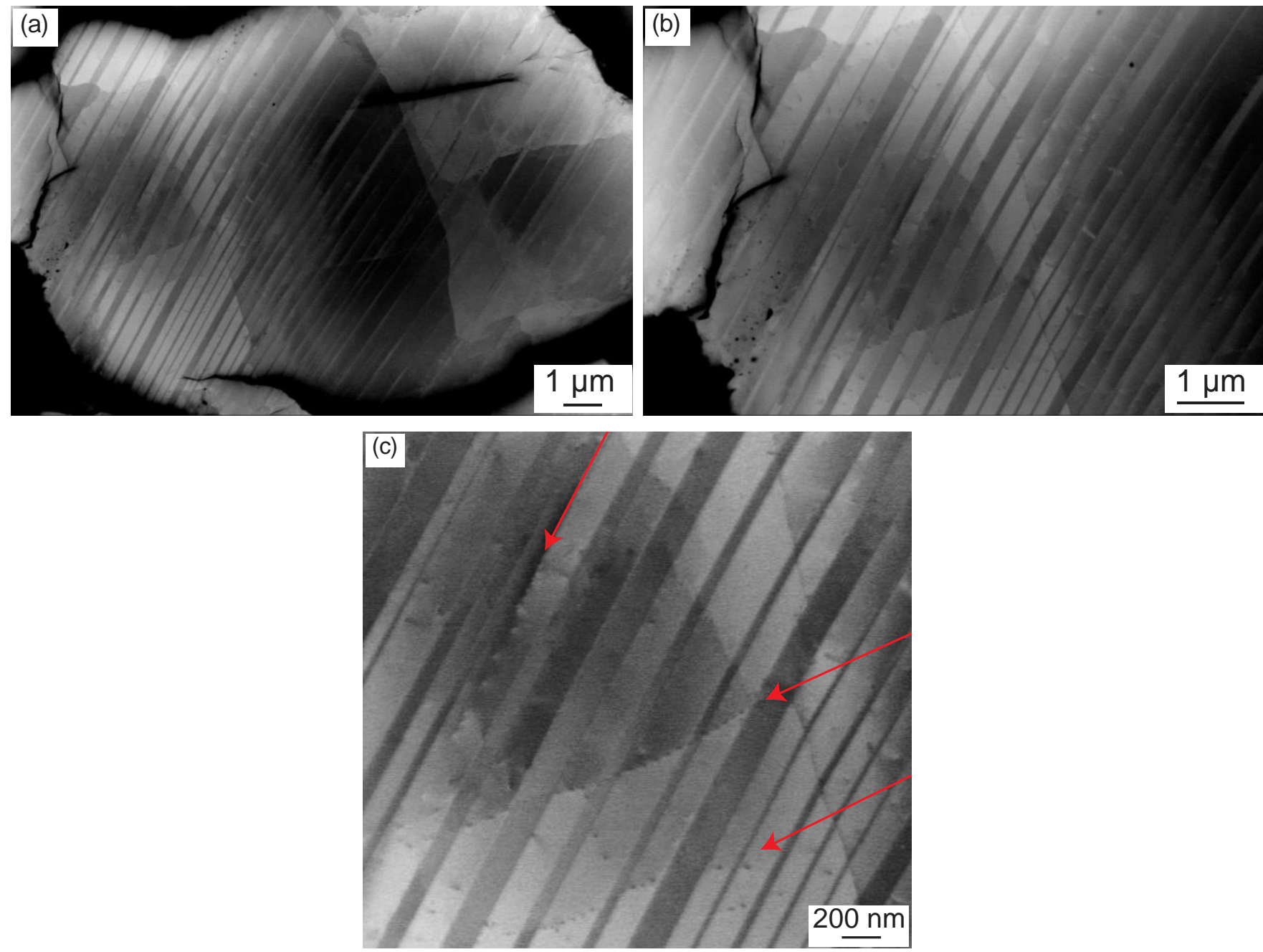

Figure 1. Electron channeling contrast imaging (ECCI) on polycrystalline diopside $\left(\mathrm{MgCaSi}_{2} \mathrm{O}_{6}\right)$ bulk sample hot pressed and deformed in a Griggs apparatus. (a) Deformed grain containing twins and subgrain boundaries, (b) and (c) higher magnification view of edge-on dislocations decorating twin boundaries and constructing subgrain boundaries indicated by arrows in (c). 